\title{
Medical Image of the Week: Neurogenic Pulmonary Edema
}
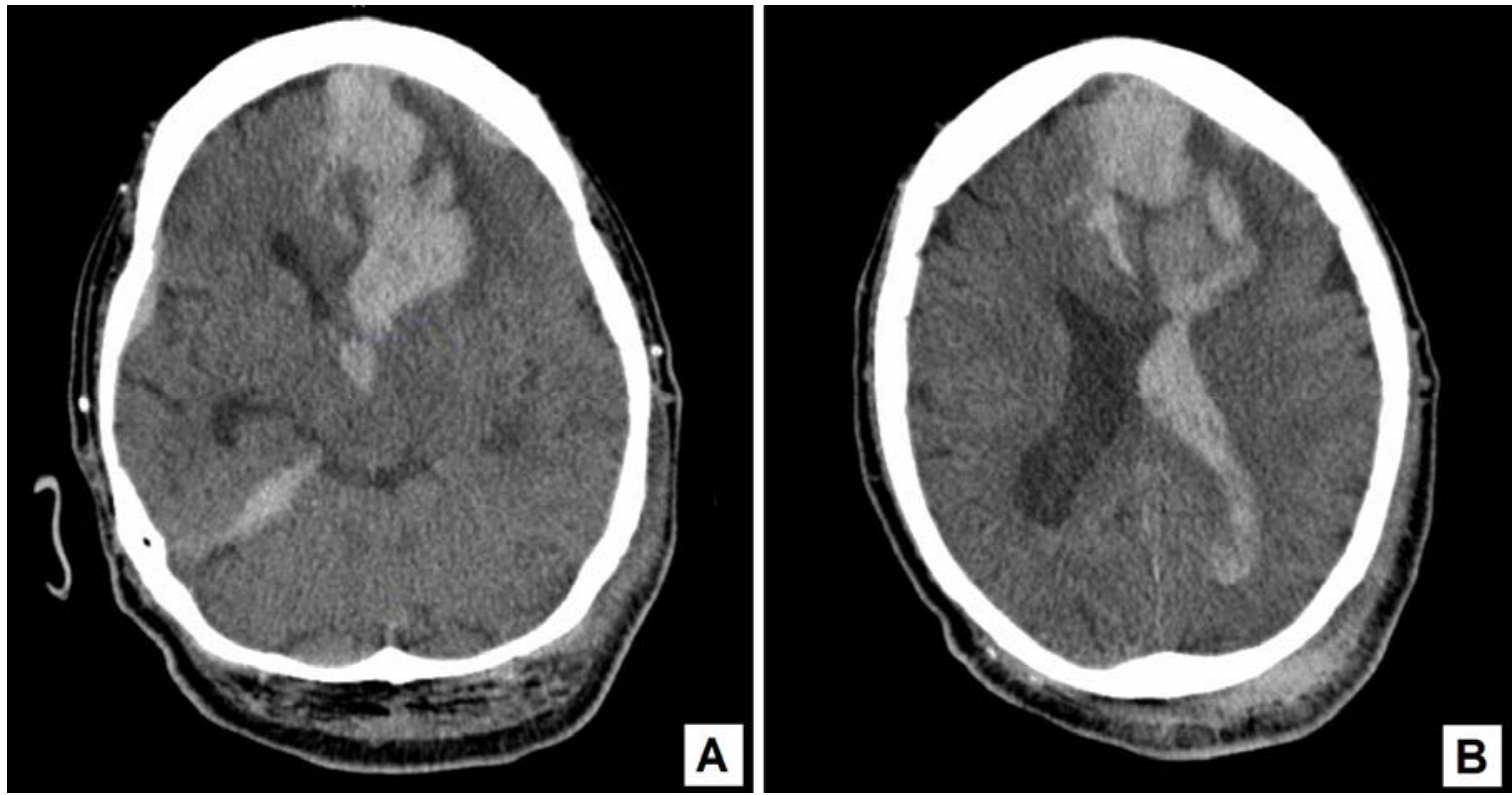

Figure 1. CT scan showing intraparenchymal hemorrhage in the left frontal lobe, scattered subdural, subarachnoid and intraventricular hemorrhage with $1.1 \mathrm{~cm}$ of left to right midline shift

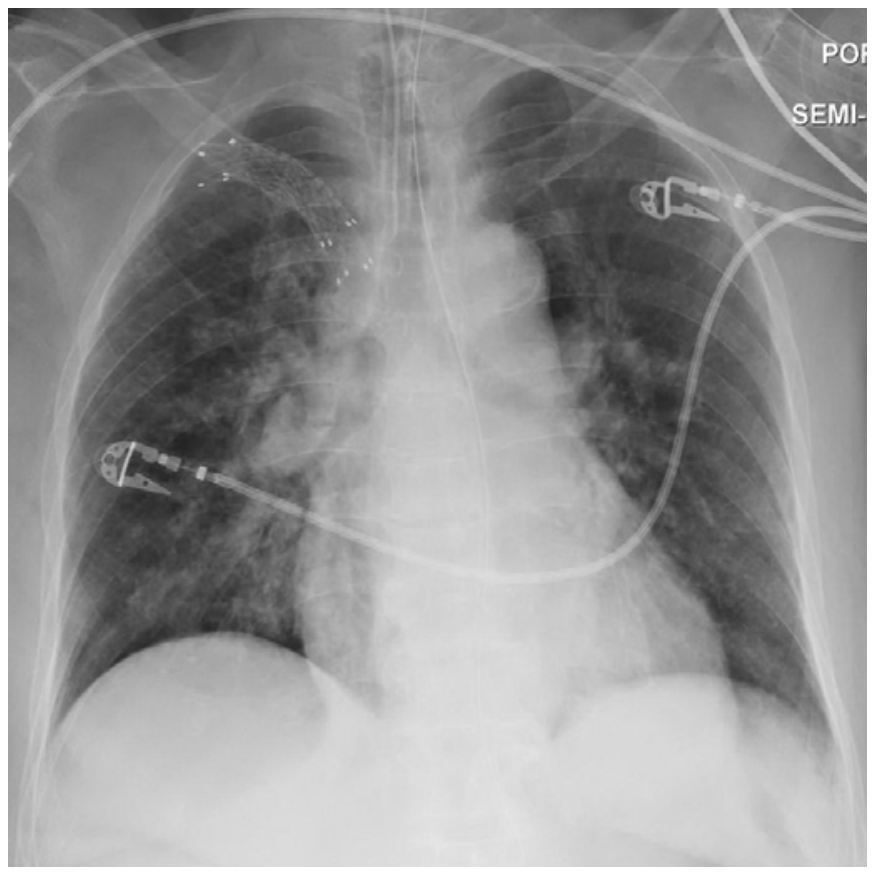

Figure 2. Chest X-ray showing central vascular congestion with bilateral pulmonary edema throughout the lung fields 
A 79 year old woman with a history of diabetes, hypertension and subarachnoid hemorrhage presented to the emergency department (ED) with altered mental status. The patient had a fall one day prior to admission, and hit her head on the ground. There was no loss of consciousness or seizure activity at that moment, however, she was found unresponsive in the bathroom the next day with brownish vomitus in the mouth and on her face. CT of the head without contrast showed a large intraparenchymal hemorrhage on the left frontal lobe with subdural hemorrhage on the right frontal and temporal lobe. Also, intraventricular blood with $1.1 \mathrm{~cm}$ left to right midline shift was observed (Figure 1). Although she had no history of left heart failure or pulmonary disease, physical exam showed coarse lung sound and chest X-ray showed acute change with prominence central vasculature with fluffy central airspace opacities, which were consistent with neurogenic pulmonary edema secondary to intracranial hemorrhage (Figure 2). An external ventricular drain was placed by neurosurgery and patient was intubated for airway protection, however she passed away the next day after her family decided comfort care.

The most common cause of neurogenic pulmonary edema is central nervous system injury including cerebral hemorrhage, head trauma and epileptic seizure (1). It usually develops several hours after an insult, although cases of immediate or delayed onset have been reported. The most common symptoms and signs include dyspnea, hemoptysis, tachypnea, tachycardia, which are not secondary to heart or lung parenchymal disease. Aspiration pneumonia is common presentation of patients with altered mental status, and it is hard to differentiate neurogenic pulmonary edema from aspiration pneumonia, however neurogenic pulmonary edema tends to develop and resolve more rapidly with no signs of infection such as fever and focal infiltration (2). The prognosis of neurogenic pulmonary edema mainly depends on the neurologic pathology rather than pulmonary edema itself, and the mainstream of treatment is supportive care, although medications including $\beta$-agonists, dobutamine or chlorpromazine can be tried.

Seongseok Yun, MD PhD; Tuan Phan, MD; Natasha Sharda, MD Department of Medicine, University of Arizona, Tucson, AZ 85724, USA

\section{References}

1. Neurogenic pulmonary oedema. Lancet. 1985;1(8443):1430-1. [PubMed]

2. Colice GL, Matthay MA, Bass E, Matthay RA. Neurogenic pulmonary edema. Am Rev Respir Dis. 1984;130(5):941-8. [PubMed] 